\title{
Clinical utility gene card for: Johanson-Blizzard syndrome
}

\author{
Maja Sukalo ${ }^{1}$, Julia Mayerle ${ }^{2}$ and Martin Zenker ${ }^{\star, 1}$ \\ European Journal of Human Genetics (2014) 22, doi:10.1038/ejhg.2013.65; published online 8 May 2013
}

\section{DISEASE CHARACTERISTICS}

1.1 Name of the disease (synonyms)

Johanson-Blizzard syndrome (JBS), Nasal alar hypoplasia, hypothyroidism, pancreatic achylia and congenital deafness.

\subsection{OMIM\# of the disease \\ 243800 .}

1.3 Name of the analysed genes or DNA/chromosome segments UBR1.

\subsection{OMIM\# of the gene(s)}

605981.

\subsection{Mutational spectrum}

Fifty-nine different mutations are known (including published mutations $^{1-8}$ and unpublished mutations identified in our lab). These include nonsense mutations (15), splice site mutations (14), small deletions and duplications/insertions causing frameshift (9), small inframe deletions (3) and missense mutations (18). Whole-gene deletions or exon deletions/duplications would be plausible mutations but have not been reported or found in our lab, so far. No obvious mutational hotspots or common mutations are seen, except for some clustering of missense mutations in the highly conserved UBR1 box.

The majority of mutations are unique within families, a few are recurrent ones. No obvious predominant founder alleles are known, so far, for certain populations.

In some cases, milder phenotypes have been found to be associated with missense mutations or small in-frame deletions, suggesting that at least some of these mutations may represent hypomorphic alleles. ${ }^{7}$ This does not allow the conclusion that missense mutations generally lead to a less-severe clinical expression!

Mutations in affected individuals occur in either homozygous or compound-heterozygous state.

\subsection{Analytical methods}

Sanger sequencing of all 47 UBR1 exons and the flanking intronic regions is the screening method that has been used for the detection of currently known UBR1 mutations. Oligonucleotide sequences for PCR primers have been published. ${ }^{1}$ Multiplex ligation-dependent probe amplification (MLPA) to screen for exon deletions/duplications is available at our centre (no purchasable MLPA kit is currently available). Analysis of microsatellites flanking the UBR1 locus may be used for detection or exclusion of homozygosity at/linkage to the UBR1 locus.

\subsection{Analytical validation}

Clinical relevance of the presumed pathogenic mutation (eg, in the case of novel missense alterations or variations with possible impact on splicing) is verified by analysis of independent control samples for this variation, comparison with database entries, use of in silico prediction methods and segregational analysis of the family. cDNA sequencing and immunoblotting may also be performed to study the consequences of the variation on splicing and UBR1 protein expression, respectively. The latter methods are not available in the diagnostic routine.

1.8 Estimated frequency of the disease (incidence at birth ('birth prevalence') or population prevalence)

The precise frequency of the disease or of UBR1 mutation carriers is not known. On the basis of an epidemiological study on anorectal anomalies in Europe, ${ }^{9}$ a prevalence of roughly 1 in 250000 life births was estimated. ${ }^{1}$ So far, no significant or ethnic differences of prevalence have been observed. Because of the autosomal-recessive mode of inheritance, a significant proportion of patients affected by JBS originate from populations with a higher frequency of consanguineous marriages.

1.9 If applicable, prevalence in the ethnic group of investigated person

Not applicable.

\subsection{Diagnostic setting}

\begin{tabular}{lcc}
\hline & Yes. & No. \\
A. (Differential) diagnostics & $\bigotimes$ & $\square$ \\
B. Predictive testing & $\square$ & $\square$ \\
C. Risk assessment in relatives & $\bigotimes$ & $\square$ \\
D. Prenatal & $\bigotimes$ & $\square$
\end{tabular}

\section{Comment:}

Mutation testing in the UBR1 gene is conducted to confirm a clinical diagnosis of JBS. Families with proven UBR1 mutations can be offered prenatal and carrier testing. As clinical signs of JBS are usually

${ }^{1}$ Institute of Human Genetics, University Hospital Magdeburg, Magdeburg, Germany; ${ }^{2}$ Department of Medicine A, University Medicine, Ernst-Moritz-Arndt-University Greifswald, Greifswald, Germany

*Correspondence: Professor M Zenker, Institute of Human Genetics, University Hospital Magdeburg, Leipziger Str. 44, Magdeburg 39120 , Germany. Tel: +49 391 6715062;

Fax: +49 391 6715066; E-mail: martin.zenker@med.ovgu.de 
present from birth, the genetic test, even in young children, is to be considered diagnostic and not predictive.

\section{TEST CHARACTERISTICS}

\begin{tabular}{|c|c|c|c|c|}
\hline & \multicolumn{2}{|c|}{ Genotype or disease } & \multirow{2}{*}{$\begin{array}{l}\text { A: True positives } \\
\text { B: False positives }\end{array}$} & \multirow{2}{*}{$\begin{array}{l}\text { C: False negative } \\
\text { D: True negative }\end{array}$} \\
\hline & Present & Absent & & \\
\hline \multicolumn{5}{|l|}{ Test } \\
\hline \multirow[t]{2}{*}{ Positive } & $A$ & B & Sensitivity: & $A /(A+C)$ \\
\hline & & & Specificity: & $D /(D+B)$ \\
\hline \multirow[t]{2}{*}{ Negative } & $\mathrm{C}$ & $\mathrm{D}$ & Positive predictive value: & $A /(A+B)$ \\
\hline & & & Negative predictive value: & $D /(C+D)$ \\
\hline
\end{tabular}

2.1 Analytical sensitivity

(proportion of positive tests if the genotype is present)

Nearly $100 \%$.

\subsection{Analytical specificity}

(proportion of negative tests if the genotype is not present)

Nearly $100 \%$.

\subsection{Clinical sensitivity}

(proportion of positive tests if the disease is present)

The clinical sensitivity can be dependent on variable factors, such as age or family history. In such cases, a general statement should be given, even if a quantification can only be made case by case. Over $95 \%$.

There is so far no evidence for genetic heterogeneity of JBS. Among 49 unrelated families with a well-founded clinical diagnosis of JBS, we have failed to identify the disease-causing mutation on only two alleles ( $\sim 2 \%$ of disease-associated alleles). Because JBS-associated mutations cause loss of function, it can be assumed that mutations may exist that remain undetected by sequencing of exons and adjacent intronic regions as well as by MLPA (eg, promoter mutations and far intronic mutations). In those cases where no UBR1 mutations can be identified on one or both alleles, mRNA analysis could be an extra possibility to detect a causative (intronic) mutation.

\subsection{Clinical specificity}

(proportion of negative tests if the disease is not present)

The clinical specificity can be dependent on variable factors such as age or family history. In such cases, a general statement should be given, even if a quantification can only be made case by case.

$100 \%$.

On the basis of current experience, it can be excluded that a healthy individual carries disease-causing UBR1 mutations on both alleles.

\subsection{Positive clinical predictive value}

(life-time risk of developing the disease if the test is positive). $100 \%$

Penetrance of the disease is complete, and in all known cases symptoms had been present from birth. There is, however, considerable variability in the clinical expression with a larger interfamilial than intrafamilial variability. Because genotype-phenotype correlations are only tentative (see 1.5 ), genotype-based predictions regarding severity of the disease are very limited.
2.6 Negative clinical predictive value

(probability of not developing the disease if the test is negative).

Assume an increased risk based on family history for a non-affected person. Allelic and locus heterogeneity may need to be considered.

Index case in that family had been tested:

Nearly $100 \%$

If disease-causing UBR1 mutations (or alleles) in the index case are known, exclusion of at least one of these mutations (or alleles) is sufficient to rule out the disease in a sibling.

Index case in that family had not been tested:

Over 95\% (assuming lack of genetic heterogeneity and a clinical sensitivity of the test of over $95 \%$ ).

\section{CLINICAL UTILITY}

3.1 (Differential) diagnostics: The tested person is clinically affected

(To be answered if in 1.10 'A' was marked)

\subsubsection{Can a diagnosis be made other than through a genetic test?}

\begin{tabular}{lll}
\hline No. & $\square$ (continue with 3.1.4) \\
Yes, $\quad$ Clinically & $\square$ \\
& Imaging & $\square$ \\
& Endoscopy & $\square$ \\
& Biochemistry & $\square$ \\
& Electrophysiology & $\square$ \\
& Other (please describe) & Audiometry and thyroid hormones
\end{tabular}

3.1.2 Describe the burden of alternative diagnostic methods to the patient

Clinical suspicion of the diagnosis is mainly based on clinical examination and history that do not represent any relevant burden to the patient. In the majority of patients, the clinical picture is very clear and distinctive - at least for someone who is familiar with this rare condition. Stool tests (fat excretion and fecal elastase determination), imaging by ultrasound/CT and rarely endoscopy may be required to substantiate exocrine pancreatic insufficiency. Furthermore, audiometry, blood tests of thyroid hormones and dental X-rays are helpful to corroborate the diagnosis. These examinations are also required, if the diagnosis is made by genetic testing, in order to realize the full range of clinical manifestations.

3.1.3 How is the cost effectiveness of alternative diagnostic methods to be judged?

Clinical, apparative and laboratory examinations as described in 3.1.2 do not really cause extra costs, as these diagnostic tests have to be recommended anyway in order to guide treatment measures. Genetic testing, however, is the only diagnostic tool that provides a definite diagnosis, and thus cannot be replaced by alternative methods in cases where some uncertainty remains about the clinical diagnosis. Moreover, identification of the causative mutations in a family is the precondition for carrier identification or early prenatal testing.

\subsubsection{Will disease management be influenced by the result of} a genetic test?
No. $\square$
Yes. $\otimes$ 
Therapy (please describe)

Prognosis (please describe)

Management

(please describe)
As treatment of JBS is purely symptomatic, all therapeutic measures mainly depend on the individual clinical problems and not on the genetic test result. Treatment may include pancreatic enzyme replacement, surgery for correction of congenital malformations, hearing aids, thyroid hormone substitution and so on.

Because known genotype-phenotype correlations are only tentative, results of genetic testing are unlikely to modify the prognosis. However, the molecular test is essential to confirm the clinical diagnosis and for accurate genetic counselling of the families concerned. The possibility of molecular carrier enables more precise genetic risk assessment in family members. Although no standards for the management of JBS exist so far, the confirmation of this diagnosis by molecular genetic testing points out medical issues that have to be considered during follow-up, eg, dental problems (usually not present in primary teeth), late development of deafness or diabetes ${ }^{10}$ and so on. Multidisciplinary follow-up covering all the health issues known in JBS has to be recommended.
3.2 Predictive setting: The tested person is clinically unaffected but carries an increased risk based on family history

(To be answered if in 1.10 ' $\mathrm{B}$ ' was marked)

\subsubsection{Will the result of a genetic test influence lifestyle and prevention?}

If the test result is positive (please describe)

Not applicable.

If the test result is negative (please describe)

Not applicable.

3.2.2 Which options in view of lifestyle and prevention does a person at-risk have if no genetic test has been done (please describe)?

Not applicable.

3.3 Genetic risk assessment in family members of a diseased person (To be answered if in 1.10 ' $\mathrm{C}$ ' was marked)

\subsubsection{Does the result of a genetic test resolve the genetic situation in that family?}

Usually yes. The parents of the index are assumed to be heterozygous carriers for the disease. Healthy siblings or other healthy relatives can be tested for their carrier status, if this is relevant for reproductive decisions. A positive genetic test confirms the autosomal-recessive mode of inheritance in the family.

\subsubsection{Can a genetic test in the index patient save genetic or other tests in family members?}

Not fully, however, identification of the causative mutations in the index case allows a very focussed genetic testing in family members instead of complete screening of the entire gene.

\subsubsection{Does a positive genetic test result in the index patient enable a predictive test in a family member?}

Predictive testing is not applicable. Only carrier status can be tested.

\subsection{Prenatal diagnosis}

(To be answered if in 1.10 'D' was marked)
3.4.1 Does a positive genetic test result in the index patient enable a prenatal diagnosis?

Yes, it provides a possibility of very early and reliable prenatal testing or even preimplantation genetic diagnosis.

\section{IF APPLICABLE, FURTHER CONSEQUENCES OF TESTING}

Please assume that the result of a genetic test has no immediate medical consequences. Is there any evidence that a genetic test is nevertheless useful for the patient or his/her relatives? (Please describe).

The result of the molecular genetic test may have no immediate medical consequences for the affected individuals and their families, but having a positive molecular genetic diagnosis will influence genetic counselling and may influence reproductive decisions. It is likely that relatives will consider genetic counselling and carrier testing to assess their own risks. Patients themselves may benefit from the confirmation of a diagnosis that is not certain on a clinical basis alone in that unnecessary additional diagnostic tests can be saved. Moreover, possible symptoms that might occur during the course of the disease, such as oligodontia of permanent teeth, hypothyroidism, hearing impairment or diabetes are more likely to be detected early, if the diagnosis is clear. As in most genetic syndromes, confirmation of the diagnosis by molecular analysis usually greatly facilitates the acceptance of the disorder for the child and the parents, which can have important psychological benefits.

\section{CONFLICT OF INTEREST}

The authors declare no conflict of interest.

\section{ACKNOWLEDGEMENTS}

This work was supported by EuroGentest2 (Unit 2: 'Genetic testing as part of health care'), a Coordination Action under FP7 (Grant Agreement Number 261469) and the European Society of Human Genetics and by the German Research Foundation (DFG) grant ZE 5242-3 to M.Z. and grant MA 4115/1-3 to J.M.

1 Zenker M, Mayerle J, Lerch MM et al: Deficiency of UBR1, a ubiquitin ligase of the $\mathrm{N}$-end rule pathway, causes pancreatic dysfunction, malformations and mental retardation (Johanson-Blizzard syndrome). Nat Genet 2005; 37: 1345-1350.

2 Al-Dosari MS, Al-Muhsen S, Al-Jazaeri A, Mayerle J, Zenker M, Alkuraya FS: JohansonBlizzard syndrome: report of a novel mutation and severe liver involvement. Am J Med Genet A 2008; 146A: 1875-1879.

3 Elting M, Kariminejad A, de Sonnaville ML et al: Johanson-Blizzard syndrome caused by identical UBR 1 mutations in two unrelated girls, one with a cardiomyopathy. $A m \mathrm{~J}$ Med Genet A 2008; 146A: 3058-3061.

4 Alkhouri N, Kaplan B, Kay M et al: Johanson-Blizzard syndrome with mild phenotypic features confirmed by UBR1 gene testing. World J Gastroenterol 2008; 14: 6863-6866

5 Fallahi GH, Sabbaghian M, Khalili M, Parvaneh N, Zenker M, Rezaei N: Novel UBR1 gene mutation in a patient with typical phenotype of Johanson-Blizzard syndrome. Eur J Pediatr 2011; 170: 233-235.

6 Schoner K, Fritz B, Huelskamp G et al: Recurrent Johanson-Blizzard syndrome in a triplet pregnancy complicated by urethral obstruction sequence: a clinical, molecular, and immunohistochemical approach. Pediatr Dev Pathol 2012; 15: 50-57.

7 Hwang CS, Sukalo M, Batygin $O$ et al: Ubiquitin ligases of the $\mathrm{N}$-end rule pathway: assessment of mutations in UBR1 that cause the Johanson-Blizzard syndrome. PLoS One 2011; 6: e24925.

8 Almashraki N, Abdulnabee MZ, Sukalo M, Alrajoudi A, Sharafadeen I, Zenker M: Johanson-Blizzard syndrome. World J Gastroenterol 2011; 17: 4247-4250.

9 Cuschieri AEUROCAT Working Group. Anorectal anomalies associated with or as part of other anomalies. Am J Med Genet 2002; 110: 122-130.

10 Nagashima K, Yagi H, Kuroume T: A case of Johanson-Blizzard syndrome complicated by diabetes mellitus. Clin Genet 1993; 43: 98-100 